\title{
Escala Brasileira de Solidão: Análises de Resposta ao Item e definição dos pontos de corte
}

\author{
Brazilian Loneless Scale: Item Response Analysis
}

and definition of cutoff points

Sabrina Martins Barroso', Valéria Sousa de Andrade', Nadyara Regina de Oliveira'

\section{RESUMO}

Objetivo: Este estudo investigou as características dos itens da Escala Brasileira de Solidão UCLA (UCLA-BR) e buscou definir os pontos de corte do instrumento para o contexto brasileiro pela Teoria de Resposta ao Item. Métodos: Utilizou-se o modelo de Resposta Gradual Politônica nos 20 itens da UCLA-BR, respondidos por 1.438 participantes de seis estados brasileiros. Estimou-se a capacidade de discriminação e dificuldade dos itens. Estimaram-se os pontos de corte para a escala. Resultados: A UCLA-BR mostrou boa capacidade para fornecer informações sobre a solidão. Dezesseis itens mostraram capacidade de dis-

\section{Palavras-chave}

Solidão, Teoria da Resposta ao Item, ponto de corte, UCLA-BR.

\section{Keywords}

Loneliness, Item Response Theory, cutoff point, UCLA-BR. criminação alta ou muito alta e quatro mostraram discriminação moderada. Os pontos de corte foram definidos. Conclusões: A UCLA-BR mostrou bons índices psicométricos e a Teoria de Resposta ao Item possibilita avanços na análise das qualidades psicométricas de instrumentos.

\section{ABSTRACT}

Objective: This study aimed to study the characteristics of the items of Brazilian Loneliness Scale UCLA (UCLA-BR) and define its the cutoff points to the Brazilian context by the Item Response Theory. Methods: It was used the Politonic Graded Response model on the 20 items of UCLA-BR answered by 1,438 participants in six states in Brazil. The discrimination capacity and item difficulties were estimated as well as the scale cutoff points. Results: UCLA-BR showed to have good capacity to provide information about loneliness. Sixteen items had high or very high discrimination capacity and four showed moderate discrimination. The cutoff points were defined. Conclusions: UCLA-BR showed good psychometric indexes. The Item Response Theory made possible the analysis of psychometric qualities of instruments.

1 Universidade Federal do Triângulo Mineiro (UFMT).

Endereço para correspondência: Sabrina Martins Barroso Rua Carmelita Rezende, 70, bloco F, ap. 03, Parque do Mirante 38081-480 - Uberaba, MG, Brasil Telefones: (34) 9917-0850/(34) 3317-4260

E-mail:smb.uftm@gmail.com 


\section{INTRODUÇÃO}

A solidão é definida como uma percepção cognitiva de que os relacionamentos sociais existentes na vida de alguém são insuficientes ou inadequados, gerando uma reação afetiva de tristeza e vazio'. Isso significa que uma pessoa vivencia a solidão caso venha a se sentir triste ou vazia por não haver pessoas por perto ou se, mesmo na presença de pessoas, não se sentir emocionalmente conectada a ninguém ${ }^{1-3}$.

Investigações realizadas na Europa e nos Estados Unidos têm indicado grande variação na prevalência da solidão entre países e faixas etárias. Uma investigação ${ }^{4} \mathrm{com}$ pessoas acima de 65 anos, realizada em 12 países europeus, indicou prevalência de solidão entre 25\% na Dinamarca e 60\% na Grécia. Nos Estados Unidos um estudo ${ }^{5}$ indicou prevalência da solidão entre 15\% e 30\% para tal população, enquanto uma metanálise ${ }^{1}$ indicou que $80 \%$ das pessoas abaixo de 18 anos e 40\% das acima de 65 anos relataram sentir solidão pelo menos algumas vezes por semana.

Além disso, um estudo realizado com crianças com idades entre 5 e 10 anos $^{3}$ mostrou que algumas delas já sentem solidão e que tal sentimento gera impacto negativo na capacidade de adaptação e desempenho escolar das crianças. Entre os adultos e idosos tem sido observado que pessoas que se percebem como mais solitárias apresentam risco aumentado para depressão, para ideação e comportamento suicida, além de maior aceleração do declínio cognitivo ${ }^{4-6}$.

Uma busca realizada nas bases de dados PubMed, Lilacs e SciELO, por artigos sobre a prevalência da solidão na população brasileira, publicados entre 2000 e 2015, não identificou nenhum resultado. Foram localizados 104 trabalhos sobre solidão publicados, mas, ao se refinar a análise considerando os objetivos dos artigos, observou-se que nenhum focava na identificação da prevalência da solidão no Brasil. Um dos pontos que pode ter dificultado a realização desse tipo de estudo foi a ausência de instrumentos validados para mensurar a solidão. Essa dificuldade foi sanada quando a Escala Brasileira de Solidão UCLA (UCLA-BR) foi validada para brasileiros com idade entre 18 e 87 anos. Contudo, em sua validação não foram definidos pontos de corte para o instrumento nem identificada a capacidade discriminativa de seus itens ${ }^{7}$. Nesse sentido, a aplicação da Teoria de Resposta ao Item (TRI) pode ser útil para conhecer melhor as características da UCLA-BR, definir seus pontos de corte e ampliar sua utilização por profissionais de saúde e pesquisadores no Brasil.

A TRI utiliza modelos lineares generalizados em suas análises, o que permite identificar características de cada item do teste/escala, e não apenas do instrumento todo ${ }^{8-11}$, de forma a apreender a relação entre as respostas aos itens e o traço latente, permitindo pensar em novos usos e composições dos testes ${ }^{8-11}$. A TRI também apresenta uma forma alternativa para definição de pontos de corte, baseando-se no nível de discriminação máximo do instrumento ${ }^{10-12}$. Considerando a recente disponibilização da UCLA-BR para uso por profissionais de saúde no Brasil e o desconhecimento sobre a contribuição individual de seus itens para a compreensão da solidão, o presente trabalho visou analisar o instrumento por meio da TRI para identificar as características discriminativas dos itens e definir os pontos de corte para utilização da escala.

\section{MÉTODOS}

\section{Participantes}

Participaram do estudo 1.438 pessoas, de ambos os sexos, com idade entre 20 e 87 anos, residentes nos estados de Minas Gerais, São Paulo, Espírito Santo, Rio Grande do Sul, Goiás e Tocantins. Entre os participantes, $66 \%$ eram mulheres, 63,80\% tinham escolaridade entre ensino médio completo e superior completo, 53\% eram solteiros e $56,70 \%$ se consideraram solitários às vezes.

\section{Instrumento}

Escala Brasileira de Solidão UCLA (UCLA-BR): escala adaptada e validada para o Brasil por Barroso, Andrade, Midgett e Carvalho ${ }^{7}$, da escala norte-americana original desenvolvida por Russel, Peplau e Cutrona ${ }^{13}$. É composta por 20 afirmações sobre sentimentos ou ações ligadas à solidão. As alternativas de resposta, em escala likert de quatro pontos, variam entre 0 (nunca) e 3 (frequentemente). A pontuação máxima do instrumento é de 60 pontos.

\section{Procedimentos para coleta dos dados}

Os participantes responderam ao instrumento presencialmente ou por meio do site de coleta de dados "SurveyMonkey". O projeto foi aprovado pelo Comitê de Ética em Pesquisa da Universidade Federal do Triângulo Mineiro, sob o Protocolo no 2.664/2013. Todos os participantes assinaram o Termo de Consentimento Livre ou deixaram sua concordância registrada no site de coleta.

\section{Análise de dados}

Inicialmente, realizaram-se análises descritivas de frequência e tendência central. Para aplicar as análises TRI do modelo de dois parâmetros para itens politômicos, é necessário demonstrar os parâmetros de unidimensionalidade do traço latente, com um traço explicando uma variância mínima de $20 \%$ e independência local estocástica ${ }^{8,10,11}$. Neste estudo, tais parâmetros foram avaliados por análise fatorial exploratória pelo método dos componentes principais, considerando nível de significância de 5\% e análise da contribuição de componentes, pela divisão engenvalue pelo menor engenvalue, como proposto por Castro et al. ${ }^{12}$. 
As análises de discriminação individual dos itens (a), nível de dificuldade (b) e da quantidade de informação fornecida sobre o traço latente $(\theta)$ foram feitas por meio de modelos de dois parâmetros para escalas politônicas. $O$ parâmetro $a$ indica quanto um item é capaz de diferenciar indivíduos com base na quantidade específica do traço latente, por meio de curvas características dos itens. A capacidade de discriminação é ordenada de forma que itens $\operatorname{com} a=0$ não possuem capacidade de discriminação, itens entre 0,01 e 0,34, possuem discriminação muito baixa, itens entre 0,35 e 0,64 indicam discriminação baixa, itens entre 0,65 e 1,34 indicam discriminação moderada, itens entre 1,35 e 1,69 mostram discriminação alta e itens com parâmetro a de 1,70 ou mais mostram capacidade de discriminação muito alta'11,12.

O parâmetro de dificuldade (b) indica os itens em que os indivíduos têm maior chance de responder corretamente ou, no presente caso, a chance de responder diante da maior intensidade da solidão. Tal parâmetro varia entre -3,0 e 3,0, e os maiores valores positivos indicam itens com maior dificuldade e/ou mais carregados de $\theta$.

A definição dos pontos de corte foi feita multiplicando o melhor ponto de discriminação identificado pela TRI pelo valor do desvio-padrão do instrumento total e somado com a média de pontuação no instrumento total, como proposto por alguns autores ${ }^{8,12}$. Após essa definição, ao valor da pontuação de corte foi acrescido ou subtraído o valor do desvio-padrão, para identificação dos pontos de corte secundários. Todas as análises foram conduzidas nos programas SPSS, versão 17.0, e no programa Parscale, versão 4.1.

\section{RESULTADOS}

A pontuação total da UCLA-BR variou de 0 a 55 pontos, com média de 15,87 pontos (DP $\pm 12,11$ ). As afirmações "Eu sinto que ninguém me conhece realmente bem" e "Eu me sinto infeliz por fazer tantas coisas sozinho(a)" receberam maior porcentagem de respostas "frequentemente" (Tabela 1).

A análise fatorial mostrou que a escala suportou até três fatores, com predominância de um fator, que explicou 49,43\% da variância (Tabela 2). Ao fazer o cálculo da unidimensionalidade, observou-se que o primeiro componente contribuía em 5,56 vezes para a variância do segundo componente e em 9,88 vezes para o terceiro. Com esse resultado, pode-se considerar que a suposição de unidimensionalidade foi cumprida. Alguns autores ${ }^{11}$ indicam que a independência dos itens, também necessária para seguir com as demais análises, é demonstrada quando há unidimensionalidade, assim foi dado seguimento às demais análises.

Tabela 1. Distribuição das porcentagens e tendência central da UCLA-BR, $2015(N=1.438)$

\begin{tabular}{|c|c|c|c|c|c|}
\hline Itens & Nunca & Raramente & Algumas vezes & Frequentemente & $\begin{array}{c}\text { Mediana; média e } \\
\text { (desvio-padrão) }\end{array}$ \\
\hline Eu me sinto infeliz por fazer tantas coisas sozinho(a). & 35,10 & 36,40 & 23,50 & 4,90 & 1,$00 ; 0,98(0,88)$ \\
\hline Eu não tolero ficar tão sozinho(a). & 44,80 & 31,60 & 18,30 & 5,30 & 1,$00 ; 0,84(0,90)$ \\
\hline Eu sinto que não tenho companhia. & 40,70 & 33,80 & 21,60 & 3,90 & 1,$00 ; 0,89(0,88)$ \\
\hline Eu sinto que ninguém me compreende. & 35,70 & 35,30 & 22,90 & 6,00 & 1,$00 ; 0,99(0,91)$ \\
\hline Eu fico esperando as pessoas me ligarem ou escreverem. & 42,10 & 28,70 & 21,60 & 7,60 & 1,$00 ; 0,95(0,97)$ \\
\hline Eu sinto que não tenho ninguém a quem eu possa recorrer. & 61,00 & 23,60 & 12,40 & 3,00 & 0,$00 ; 0,57(0,82)$ \\
\hline Eu não me sinto próximo(a) a ninguém. & 59,60 & 23,50 & 14,20 & 2,70 & 0,$00 ; 0,60(0,83)$ \\
\hline Sinto que meus interesses e ideias não são compartilhados por aqueles que me rodeiam. & 38,20 & 33,70 & 22,40 & 5,70 & 1,$00 ; 0,96(0,91)$ \\
\hline Eu me sinto excluído(a). & 48,10 & 31,80 & 16,60 & 3,50 & 1,$00 ; 0,75(0,85)$ \\
\hline Eu me sinto completamente sozinho(a). & 60,20 & 25,20 & 11,70 & 2,90 & 0,$00 ; 0,57(0,81)$ \\
\hline Eu sou incapaz de me aproximar e de me comunicar com as pessoas ao meu redor. & 60,80 & 25,40 & 11,30 & 2,40 & 0,$00 ; 0,55(0,79)$ \\
\hline Eu sinto que minhas relações sociais são superficiais. & 37,90 & 34,80 & 22,70 & 4,60 & 1,$00 ; 0,94(0,89)$ \\
\hline Eu me sinto carente de companhia. & 38,80 & 33,90 & 22,30 & 5,10 & 1,$00 ; 0,94(0,90)$ \\
\hline Eu sinto que ninguém me conhece realmente bem. & 34,40 & 31,80 & 25,30 & 8,40 & 1,$00 ; 1,08(0,96)$ \\
\hline Eu me sinto isolado(a) das outras pessoas. & 47,60 & 31,80 & 17,20 & 3,40 & 1,$00 ; 0,76(0,85)$ \\
\hline Sou infeliz estando tão excluído(a). & 66,90 & 21,30 & 8,60 & 3,10 & 0,$00 ; 0,48(0,78)$ \\
\hline Para mim é difícil fazer amigos. & 50,10 & 27,30 & 18,60 & 3,90 & 0,$00 ; 0,76(0,89)$ \\
\hline Eu me sinto bloqueado(a) e excluído(a) por outras pessoas. & 58,00 & 28,00 & 11,30 & 2,80 & 0,$00 ; 0,59(0,80)$ \\
\hline Sinto que as pessoas estão ao meu redor, mas não estão comigo. & 41,90 & 33,40 & 20,30 & 4,30 & 1,$00 ; 0,87(0,88)$ \\
\hline Eu me sinto incomodado(a) em realizar atividades sozinho(a). & 45,90 & 33,80 & 15,60 & 4,70 & 1,$00 ; 0,79(0,87)$ \\
\hline
\end{tabular}


Tabela 2. Análise fatorial da UCLA-BR, 2015 ( N = 1.438)

\begin{tabular}{|c|c|c|c|c|}
\hline \multirow{2}{*}{ Itens } & \multirow{2}{*}{ E' inicial } & \multicolumn{3}{|c|}{ Análise fatorial ${ }^{2}$} \\
\hline & & F1 & F2 & F3 \\
\hline Eu me sinto infeliz por fazer tantas coisas sozinho(a). & 9,88 & 0,63 & 0,42 & $-0,02$ \\
\hline Eu não tolero ficar tão sozinho(a). & 1,56 & 0,47 & 0,69 & 0,13 \\
\hline Eu sinto que não tenho companhia. & 1,00 & 0,76 & 0,06 & $-0,14$ \\
\hline Eu sinto que ninguém me compreende. & 0,80 & 0,69 & 0,09 & $-0,30$ \\
\hline Eu fico esperando as pessoas me ligarem ou escreverem. & 0,65 & 0,62 & 0,24 & $-0,06$ \\
\hline Eu sinto que não tenho ninguém a quem eu possa recorrer. & 0,63 & 0,72 & $-0,05$ & $-0,21$ \\
\hline Eu não me sinto próximo(a) a ninguém. & 0,57 & 0,71 & $-0,21$ & $-0,20$ \\
\hline Sinto que meus interesses e ideias não são compartilhados por aqueles que me rodeiam. & 0,54 & 0,68 & $-0,20$ & $-0,23$ \\
\hline Eu me sinto excluído(a). & 0,50 & 0,80 & $-0,17$ & 0,11 \\
\hline Eu me sinto completamente sozinho(a). & 0,45 & 0,77 & $-0,05$ & $-0,05$ \\
\hline Eu sou incapaz de me aproximar e de me comunicar com as pessoas ao meu redor. & 0,42 & 0,63 & $-0,22$ & 0,50 \\
\hline Eu sinto que minhas relações sociais são superficiais. & 0,40 & 0,69 & $-0,19$ & $-0,09$ \\
\hline Eu me sinto carente de companhia. & 0,38 & 0,74 & 0,28 & $-0,09$ \\
\hline Eu sinto que ninguém me conhece realmente bem. & 0,37 & 0,68 & $-0,05$ & $-0,33$ \\
\hline Eu me sinto isolado(a) das outras pessoas. & 0,36 & 0,80 & $-0,24$ & 0,07 \\
\hline Sou infeliz estando tão excluído(a). & 0,33 & 0,75 & 0,06 & 0,19 \\
\hline Para mim é difícil fazer amigos. & 0,32 & 0,68 & $-0,28$ & 0,42 \\
\hline Eu me sinto bloqueado(a) e excluído(a) por outras pessoas. & 0,30 & 0,79 & $-0,15$ & 0,26 \\
\hline Sinto que as pessoas estão ao meu redor, mas não estão comigo. & 0,27 & 0,78 & $-0,15$ & $-0,08$ \\
\hline Eu me sinto incomodado(a) em realizar atividades sozinho(a). & 0,26 & 0,55 & 0,61 & 0,23 \\
\hline Explicação da variância & & 49,43 & 7,83 & 5,02 \\
\hline
\end{tabular}

'Valores dos engenvalues. ${ }^{2}$ Componente fatorial.

A análise da quantidade total de informação mostrou que a UCLA-BR fornece um elevado grau de informações sobre a solidão (Figura 1). A análise da capacidade de discriminação dos itens (parâmetro a) mostrou que o item com maior capacidade discriminativa foi o décimo: "Eu me sinto completamente sozinho(a)" ( $a=2,69)$ (Tabela 3 e Figura 2). Para esse item, os indivíduos com nível de solidão entre -1,0 e 0,0 apresentaram maior chance de responder "nunca". Os indivíduos com nível de solidão entre 0,1 e 1,0 mostraram mais chance de responder "raramente", enquanto indivíduos com nível de solidão entre 1,1 e 2,0 apresentavam maior probabilidade de responder "algumas vezes". Os respondentes com nível de solidão acima de 2,0 apresentaram maior chance de responder "frequentemente". Essa mesma lógica pode ser aplicada a todos os outros 19 itens da UCLA-BR.

Dezesseis itens da escala demonstraram capacidade de discriminação alta ou muito alta, enquanto quatro itens da escala (itens 2, 5, 11 e 20) mostraram capacidade de discriminação moderada. A capacidade de discriminação moderada mostra que $\mathrm{o}$ item consegue diferenciar bem pessoas com traço latente extremo, mas pode mostrar mesma probabili-

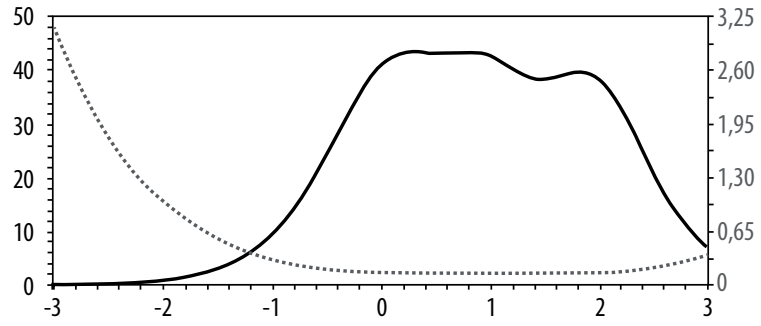

Figura 1. Informação total obtida por meio da UCLA-BR.

dade de resposta para as pessoas com traço latente no nível intermediário.

A análise do parâmetro $b$ é feita considerando o ponto na escala em que a chance da resposta mais esperada é de $50 \%{ }^{5}$. No presente estudo o item considerado mais difícil, indicativo de maior intensidade de solidão, foi o item 16 , referente a "Sou infeliz estando tão excluído(a)" ( $b=1,32)$, enquanto o item mais fácil, relacionado à menor intensidade da solidão, foi o item 14 "Eu sinto que ninguém me conhece realmente bem" $(b=0,53)$. 
Tabela 3. Estimativa dos parâmetros do Modelo de Resposta Gradual da UCLA-BR $(N=1.438)$

\begin{tabular}{|c|c|c|c|}
\hline Item & $a_{i}^{1}\left(E . P^{2}\right)$ & Discriminação & $b_{i}^{3}\left(E . P^{2}\right)$ \\
\hline Eu me sinto infeliz por fazer tantas coisas sozinho(a). & $1,39(0,04)$ & Alta & $0,66(0,04)$ \\
\hline Eu não tolero ficar tão sozinho(a). & $0,99(0,04)$ & Moderada & $0,92(0,05)$ \\
\hline Eu sinto que não tenho companhia. & $1,79(0,06)$ & & $0,74(0,04)$ \\
\hline Eu sinto que ninguém me compreende. & $1,49(0,05)$ & Alta & $0,61(0,04)$ \\
\hline Eu fico esperando as pessoas me ligarem ou escreverem. & $1,12(0,04)$ & Moderada & $0,72(0,05)$ \\
\hline Eu sinto que não tenho ninguém a quem eu possa recorrer. & $1,56(0,06)$ & Alta & $1,19(0,04)$ \\
\hline Eu não me sinto próximo(a) a ninguém. & $1,54(0,06)$ & Alta & $1,15(0,04)$ \\
\hline Sinto que meus interesses e ideias não são compartilhados por aqueles que me rodeiam. & $1,48(0,05)$ & Alta & $0,65(0,04)$ \\
\hline Eu me sinto excluído(a). & $2,15(0,09)$ & Muito Alta & $0,86(0,04)$ \\
\hline Eu me sinto completamente sozinho(a). & $2,69(0,08)$ & Muito Alta & $1,13(0,04)$ \\
\hline Eu sou incapaz de me aproximar e de me comunicar com as pessoas ao meu redor. & $1,31(0,05)$ & Moderada & $1,26(0,05)$ \\
\hline Eu sinto que minhas relações sociais são superficiais. & $1,57(0,05)$ & Alta & $0,66(0,04)$ \\
\hline Eu me sinto carente de companhia. & $1,72(0,06)$ & Muito Alta & $0,67(0,04)$ \\
\hline Eu sinto que ninguém me conhece realmente bem. & $1,37(0,04)$ & Alta & $0,53(0,04)$ \\
\hline Eu me sinto isolado(a) das outras pessoas. & $2,24(0,09)$ & Muito Alta & $0,84(0,04)$ \\
\hline Sou infeliz estando tão excluído(a). & $1,71(0,07)$ & Muito Alta & $1,32(0,04)$ \\
\hline Para mim é difícil fazer amigos. & $1,35(0,05)$ & Alta & $0,94(0,05)$ \\
\hline Eu me sinto bloqueado(a) e excluído(a) por outras pessoas. & $2,09(0,09)$ & Muito Alta & $1,08(0,04)$ \\
\hline Sinto que as pessoas estão ao meu redor, mas não estão comigo. & $2,00(0,07)$ & Muito Alta & $0,72(0,04)$ \\
\hline Eu me sinto incomodado(a) em realizar atividades sozinho(a). & $1,13(0,04)$ & Moderada & $0,91(0,05)$ \\
\hline
\end{tabular}

${ }^{1}$ Parâmetro de discriminação. ${ }^{2}$ Erro-padrão. ${ }^{3}$ Parâmetro de dificuldade.
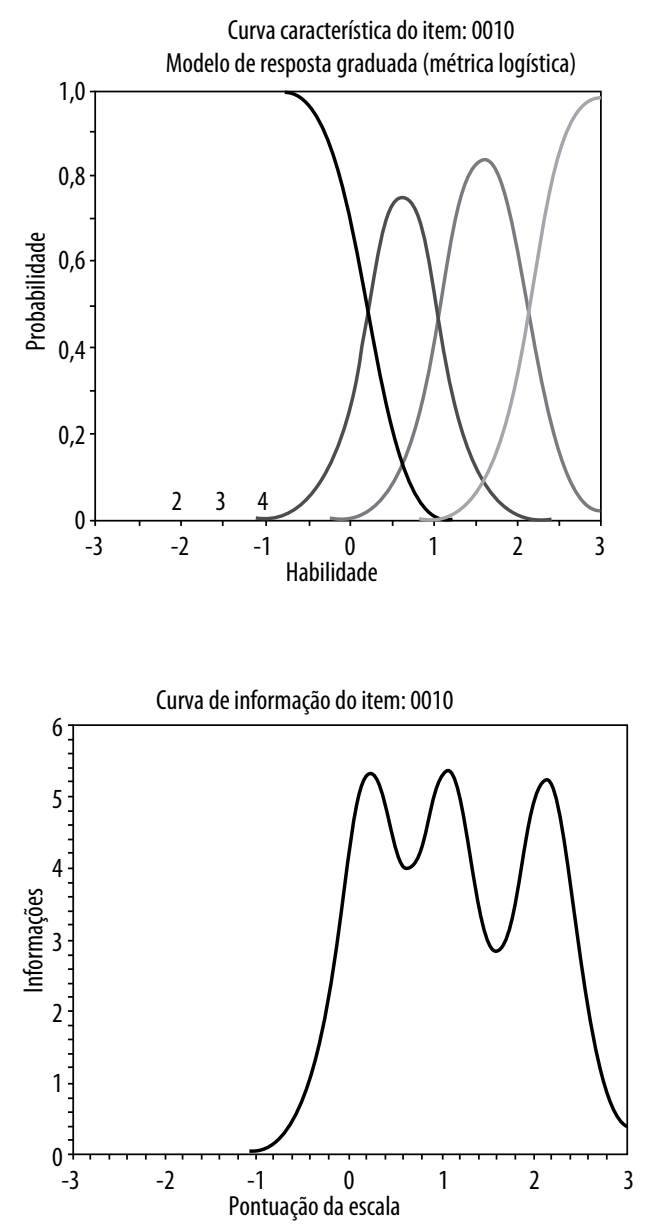

Figura 2. Curvas características do item 10 da UCLA-BR.
Após a identificação das características dos itens, prosseguiu-se com a definição dos pontos de corte do instrumento. O melhor ponto de discriminação da solidão foi de 1,61 nas análises de TRI, em um intervalo entre -3,0 e 3,0. Aplicando a fórmula de conversão ${ }^{12}$, estabeleceu-se que a pontuação mais discriminativa da escala foi a de 36 pontos. Aplicando a redução ou soma de um desvio-padrão para definição de outras intensidades de solidão, chegou-se aos seguintes pontos de corte para interpretação da UCLA-BR: 0 a 22 pontos, indicativo de solidão mínima; 23 a 35 pontos, indicativo de solidão leve; 36 a 47 pontos, indicativo de solidão moderada; e 48 a 60 pontos, indicativo de solidão intensa.

\section{DISCUSSÃO}

O objetivo deste trabalho foi analisar as qualidades psicométricas da escala UCLA-BR por meio da TRI. A escala baseia-se na definição de solidão como sentimento de vazio e tristeza gerado pela interpretação negativa por estar só ou rodeado de pessoas com quem não se possui ligação emocional ${ }^{1,2}$. Ao fazer as análises fatoriais e de TRI, percebeu-se que o instrumento consegue ser uma boa fonte de informações sobre a solidão. Esse resultado indica que o instrumento pode ser útil para profissionais que desejem mensurar a solidão de forma mais objetiva. A definição dos pontos de corte poderá, ainda, facilitar a interpretação dos resultados pelos profissionais. 
A avaliação dos itens da escala quanto à sua quantidade de informação psicométrica e ao seu grau de intensidade do traço latente é uma contribuição relevante da TRI, pois possibilita identificar qual característica ou sintoma tem maior ou menor peso na avaliação da intensidade da solidão'. Uma vantagem das análises de instrumentos pela TRI consiste em considerar essas características no cálculo do traço latente para cada respondente do instrumento, diferentemente do cálculo do escore total, em que todos os itens são considerados como igualmente importantes ${ }^{14,15}$.

Outro ganho substancial dos modelos da TRI para a interpretação de instrumentos é a geração das curvas características de resposta, as quais permitem que se observe a relação entre o nível de intensidade do construto - solidão - e a probabilidade de alguém escolher uma determinada categoria8. Tais curvas permitem identificar itens que estejam com problemas de dimensionamento e que precisem ser excluídos ou recategorizados ${ }^{12,14,15}$. No caso da UCLA-BR, não houve itens com baixo poder discriminativo, mas os resultados indicam que a recategorização dos itens 2, 5, 11 e 20 poderia aumentar sua capacidade de discriminação. Cabe destacar como limitações do trabalho a não realização das análises sugeridas por essa recategorização e a restrição da coleta de dados presencial ao estado de Minas Gerais, que limitou o acesso a pessoas que não fizessem uso da internet e residissem em outros estados à amostra.

\section{CONCLUSÕES}

Internacionalmente, as diferentes versões da Escala de Solidão UCLA têm sido bastante utilizadas para mensurar o nível de solidão em investigações científicas². Espera-se que a validação desse instrumento para o Brasil, o estabelecimento de seus pontos de corte e a demonstração de que a UCLA-BR é uma boa fonte de informações sobre a solidão aumentem a quantidade de investigações no contexto brasileiro sobre essa temática.

\section{CONTRIBUIÇÕES INDIVIDUAIS}

Sabrina Martins Barroso - Participou da idealização e execução do estudo e da preparação do artigo de divulgação dos resultados.

Valéria Sousa de Andrade - Participou da concepção do estudo e da preparação do artigo de divulgação dos resultados.
Nadyara Regina de Oliveira - Participou da coleta de dados e da preparação do artigo de divulgação dos resultados.

\section{CONFLITO DE INTERESSE}

Os autores negam conflito de interesse.

\section{AGRADECIMENTOS}

Os autores agradecem à Fundação de Amparo à Pesquisa de Minas Gerais (Fapemig) pelo apoio por meio de bolsa de iniciação científica.

\section{REFERÊNCIAS}

1. Hawkley LC, Cacioppo JT. Loneliness matters: a theoretical and empirical review of consequences and mechanisms. Ann Behav Med. 2010;40(2):1-14.

2. Russell DW, Cutrona CE, MCRae C, Gomez M. Is loneliness the same as being alone? J Psychol. 2012;146(1-2):7-22.

3. Liepins $M$, Cline T. The development of concepts of loneliness during the early years in school. School Psychol Int. 2011;32(4):397-411

4. Sundstrom G, Fransson E, Malmberg B, Davey A. Loneliness among older Europeans. Eur J Ageing. 2009;6:267-75

5. Theeke LA. Predictors of loneliness in U.S. adults over age sixty-five. Arch Psychiatr Nurs. 2009;23(5):387-96

6. VanderWeele TJ, Hawkley LC, Thisted RA, Cacioppo JT. A marginal structural model analysis for loneliness: implications for intervention trials and clinical practice. J Consult Clin Psychol. 2011;79(2):225-35.

7. Barroso SM, Andrade VS, Midgett AH, Carvalho RGN. Evidências de validade da Escala Brasileira de Solidão UCLA. J Bras Psiquiatr. 2016;65(1):68-75.

8. Pasquali L. TRI - Teoria de Resposta ao Item: Teoria, Procedimentos e Aplicações. 1.ed. Brasília: Laboratório de Pesquisa em Avaliação e Medida - LabPAM; 2007.

9. Pasquali L. Instrumentação psicológica: fundamentos e práticas. Porto Alegre: Artmed; 2010.

10. Rupp A. Item Response Modeling with BILOG-MG and MULTILOG for Windows. Int J Test. 2003;3(4):365-84.

11. Couto G, Primi R. Teoria de resposta ao item (TRI): conceitos elementares dos modelos para itens dicotômicos. Bol Psicol. 2011;61(134):1-15.

12. Castro SMJ, Trentini C, Riboldi J. Teoria da resposta ao item aplicada ao Inventário de Depressão Beck. Rev Bras Epidemiol. 2010;13(3):487-501.

13. Russell D, Peplau LA, Cutrona CE. The revised UCLA Loneliness Scale: concurrent and discriminant validity evidence. J Pers Soc Psychol. 1980;39(3):472-80.

14. McHorney CA, Cohen AS. Equating health status measures with item response theory: illustrations with functional status items. Med Care. 2000;38(9 Suppl):I143-59.

15. Baker FB. The basics of item response theory. Wisconsin: ERIC Clearinghouse on Assessment and Evaluation; 2001 